Medicine Committee, Genetics Committee. Prevention of Rh alloimmunization. $\mathrm{O}$ bstet Gynaecol Can 2003 Sep;25(9):765-773.

10. Crowther CA. Anti-D administration in pregnancy for preventing Rhesus allo immunisation (Cochrane Review). En:The Cochrane Library 2004; Issue 2. Chichester, UK: John W iley \& Sons, Ltd.

11. Baptista-G onzález H, Rosenfeld-Mann F, Leiss-Marquez T. Prevención de la isoinmunización materna al RhD con gamaglobulina anti-D. Salud Publica Mex 2001;43: 52-58.

\section{Evaluación del contenido de flúor en sal de mesa fluorada*}

Señor editor: la fluoración de la sal de mesa es un método preventivo de salud pública que se usa con éxito en varios países en el mundo ${ }^{1-4}$ para brindar a la población protección contra la caries dental. Esta medida se implementó por primera vez en algunas zonas del país en $1981^{5}$ y, posteriormente, se aplicó a escala nacional. ${ }^{6}$ Algunos estudios publicados muestran que las concentraciones de flúor $(F)$ en la sal no se encuentran dentro de los intervalos recomendados por las autoridades gubernamentales. ${ }^{3,7}$ Se han reportado diferentes concentraciones de $\mathrm{F}$ en un mismo paquete $\mathrm{o}$ bolsa. ${ }^{3,7,8}$ Por motivos de seguridad y de control de calidad se recomienda la monitorización constante de las concentraciones de F en sal. ${ }^{6}$ Algunos investigadores han propuesto que un control de calidad deficiente es el responsable del amplio intervalo de concentraciones de F que se han encontrado. Nuestro objetivo fue evaluar el contenido de $\mathrm{F}$ en muestras de la sal de mesa que se distri-

\footnotetext{
* Este estudio fue apoyado por el Grant-inAid for Minority Faculty Development (01/ 02-12/02) Intramural Grant (Office for Professional Development), de la Indiana University-Purdue University Indianapolis, USA, y por el Grant \# l-R21-DE-14716-1 del National Institute of Dental and Craniofacial Research, P.I. E.
}

buye en México, y determinar si las concentraciones de este elemento en la sal se encontraban dentro de los intervalos recomendados por las autoridades. $\mathrm{Ob}$ tuvimos una lista detallada de productores y empacadores, así como de las marcas denominadas "libre" que se comercializan en México. Estos datos se obtuvieron de páginas web, la sección amarilla y organizaciones no gubernamentales. Se contactó al principal organismo de productores de la sal, la Asociación Mexicana de la Industria de la Sal, AC (AMISAC), la cual nos proporcionó la lista de sus miembros, información sobre la producción y los nombres de las marcas disponibles de sal de mesa fluorada. Al momento de efectuar este estudio, entre enero de 2002 y marzo de 2003, en el mercado de la sal se encontraron 23 diferentes marcas que eran distribuidas por 18 productores y empacadores, así como cinco marcas "libre" de las cuatro cadenas de supermercados que las comercializan como propias. La concentración de $\mathrm{F}$ indicada en la etiqueta de estos productos era de $250 \pm 50$ partes por millón (ppm). Se obtuvieron paquetes de un $\mathrm{kg}$ de los cuales se extrajeron aproximadamente 100 gramos: 30 de la parte superior, 30 de la media y 30 de la inferior. Todas las muestras se recolectaron en México, se numeraron, empacaron y transportaron, de acuerdo con las normas internacionales, para ser analizadas en el laboratorio del Oral Hea1th Research Institute, de la Escuela de Odontología de la Universidad de Indiana, Estados Unidos de América. Para determinar la concentración de F las muestras se analizaron por medio del método de difusión. Se obtuvieron estadísticas descriptivas y coeficientes de variación, y se utilizaron pruebas de ANOVA para determinar diferencias entre las muestras.

De las 13 marcas comerciales de sal de mesa elaboradas por los productores se consiguieron cinco (39\%) y de las 10 marcas comercializadas por los empacadores ocho $(80 \%)$. Otras dos marcas se adquirieron de pequeños productores.
Asimismo, se obtuvieron cinco paquetes de sal "marca libre", las cuales equivalen a $83 \%$ de las que se comercializan en las grandes cadenas de supermercados. De esta forma, se recolectaron 18 diferentes marcas de sal fluorada, lo que representaría 95\% del mercado de la sal de mesa en México. Además de éstas se adquirieron siete duplicados de algunas marcas, para un total de 25 paquetes, que conformaron 75 muestras de sal, las cuales fueron analizadas para determinar su contenido de $\mathrm{F}$.

La concentración promedio de $\mathrm{F}$ de la sal de mesa analizada fue de 266 \pm 67 ppm F (rango 55-355 ppm). Las concentraciones promedio de las muestras por fabricante y por su localización se muestran en el cuadro I. Se efectuaron diferentes comparaciones, y no se encontraron diferencias significativas de las concentraciones de $\mathrm{F}$ en las muestras elaboradas por los productores, con las de los empacadores o con las de "marca libre". Tampoco se encontraron diferencias significativas de acuerdo con la localización de la muestra. No se hallaron diferencias entre el tipo de paquete, ya sea bolsa (234 \pm 29 ppm), bote de plástico (2 12-14) o de cartón (231£6 ppm), los cuales demostraron tener valores similares de F. Por último, las diferencias

Cuadro

Concentración Media Y DESVIACIÓN ESTÁNDAR DE MUESTRAS DE SAL FLUORADA DE PRODUCTORES, EMPACADORES Y MARCAS "LIBRE", Y POR LA LOCALIZACIÓN DE RECOLECCIÓN DE LA MUESTRA

\begin{tabular}{lcc} 
Tipo de muestra & $N=F \mu g / g \pm D E$ \\
Marcas libre & 18 & $226+45$ \\
\hline Productores & 24 & $218+64$ \\
\hline Empacadores & 33 & $229+50$ \\
$\begin{array}{l}\text { Localización de la recolección } \\
\text { Superior }\end{array}$ & \\
\hline Media & 25 & $230+49$ \\
\hline Inferior & 25 & $219+63$ \\
\hline
\end{tabular}


del análisis del contenido de $\mathrm{F}$ entre las muestras de sal de grano (222 \pm 58 ppm) y las de sal refinada (224 $\pm 32 \mathrm{ppm})$ tampoco fueron estadísticamente diferentes. Casi la mitad de las muestras de sal fluorada $(n=38)$, tuvieron concentraciones de F dentro del rango de los 250 \pm 50 ppm. Actualmente, en México se requiere por ley que la sal contenga esta concentración. ${ }^{6}$ Un tercio de las muestras tuvieron concentraciones menores a 199 ppm, seis tuvieron más de 300, mientras que otras seis tuvieron menos de 150 .

Nuestros resultados están en desacuerdo con los publicados en investigaciones previas. ${ }^{7,9}$ Por ejemplo, en el estudio conducido por Maupomé y colaboradores, en el que puntualizan las deficiencias del contenido de $\mathrm{F}$ en la sal, la mayoría de las muestras analizadas tenían muy poco F, o en exceso. Estos investigadores analizaron 221 paquetes de sal y encontraron un promedio de $48.7 \pm 69.9 \mathrm{ppm} / \mathrm{kg}$ F (intervalo $7.5 \mathrm{a}$ 475 ppm). ${ }^{7}$ Otra investigación reciente ${ }^{8}$ halló un promedio de 194 ppm F (142243 ppm). Las concentraciones de dicho estudio son $20 \%$ menores que las obtenidas en nuestro estudio. Esta diferencia quizás se deba al método utilizado para medir las concentraciones de $\mathrm{F}$ en sal. Resultados de investigaciones efectuadas por nuestro grupo han demostrado que el análisis de sal puede verse influido por la metodología que se use. ${ }^{9}$ Los resultados que mostramos indican que el control de calidad de la sal de los productores y empacadores de la sal de mesa fluorada en México ha mejorado, ya que casi la mitad se encontraba dentro del intervalo recomendado por las autoridades. No existen muchos informes de la concentración de $F$ en la sal en México; por este motivo, recomendamos la monitorización constante de la sal fluorada mediante métodos analíticos adecuados.

\section{Agradecimientos}

Agradecemos al licenciado Sergio A Moreno Huitrón, de la Asociación Mexi- cana de la Industria de la Sal (AMISAC) por su valiosa información.

E Angeles M artínez Mier, Doctor in Dental Surgery, Master of Science in Dentristy, PhD. Armando E Soto Rojas, M PH, Doctor in Dental Surgery. Christine M Buckley,

Bachelor Degree in Fine Arts George K Stookey, $A B, M S D, P h D$. Domenick T Zero,

MS, Doctor in Dental Surgery. Department of Community and Preventive Dentistry, Oral Health Research Institute, Indiana University School of Dentistry, EUA. e-mail: esmartin@ iupui.edu Jorge $M$ argineda $\mathrm{BS}, \mathrm{MS}, \mathrm{PhD}$

Department de Química, Facultad de Ciences, Universitat Autónoma de Barcelona, España.

\section{Referencias}

1. Marthaller TM, Mejía R,Tóth Viñes JJ. Cariespreventive salt fluoridation. Caries Res 1978;12 (Suppl1):15-21.

2. Bergmann KE, Bergmann RL. Salt fluoridation and general health.Adv Dent Res 1995:9(2): 138-143.

3. Estupiñán-D ay D. 0 verview of salt fluoridation in the region of the Americas: Part 1.The strategies, cost-benefit analysis, and legal mechanisms utilized in the national programs of salt fluoridation. Salt 2000, 8th W orld Salt Symposium, 2000;2: 983-988.

4. Marthaller TM, Schenardi C. Inhibition of caries in children after $51 / 2$ years use of fluoridated table salto Helv 0 dont Acta 1962;6:1-6
5. Secretaría de Salubridad y Asistencia. N orma 0 ficial Mexicana -040-SSA-1981. Reglamento de Yodatación y Fluoruración de la Sal. México, DF: Diario 0 ficial de la Federación, 26 de marzo de 1981:20-22.

6. Secretaría de Salud. N orma 0 ficial Mexicana 040-SSAI-1993. Bienes y Servicios. Sal Yodada, Sal Yodada y Fluorurada. Especificaciones Sanitarias. México, DF: Diario 0 ficial de la Federación, 13 de marzo de 1995.

7. Maupomé- $C$ arvantes $G$, Jaramillo-Lanchero RD, Andrade-D elgado LC, Juárez-Reyes $P L$, López-Pérez R, Sánchez-N avarro W, et al. Fluoride content of table salt in Mexico City. Bol 0 ficina Sanit Panam 1995;119(3):195-201. 8. Girón-A maya CN . Concentración de flúor en la sal para consumo humano distribuida en México, DF. (Tesis). México, D F: U niversidad Tecnológica de México; 1999.

9. Martínez-Mier EA, Soto-Rojas AE, U renaCirett JL, Stookey GK, D unipace AJ. Fluoride analysis of table salt samples from México. J Dent Res 2001;80:47.

\section{Solicitan corrección de error}

Señor editor: en el cuadro II del artículo "Las cesáreas en México: tendencias, niveles y factores asociados", publicado en el volumen 46, número 1 de Salud Pública de México, existe un error que puede dificultar la interpretación del mismo. El coeficiente de regresión de la constante aparece con signo negativo y los valores correspondientes a su intervalo de confianza son positivos, lo que evidentemente es una incongruencia. Por lo tanto

\section{Cuadro II \\ Resultados de LA REGRESIÓN LINEAL MÚltipLe ENTRE PORCENTAJE DE CESÁREAS Y DIFERENTES VARIABLES SOCIOECONÓMICAS. MÉXICO, 1999}

\begin{tabular}{lcc} 
Variable & Coeficiente $\beta$ & $\begin{array}{c}\text { Intervalo de confianza } \\
\text { para el coeficiente } \beta\end{array}$ \\
Constante & 14.627 & $4.23, \quad 24.47$ \\
\hline Producto interno bruto per cápita & 0.00043 & $0.000, \quad 0.001$ \\
\hline Porcentaje de médicos que son especialistas & 47.87 & $12.55,83.29$
\end{tabular}

$\mathrm{R} 2=0.528$

Variables excluidas: porcentaje de madres con estudios profesionales, porcentaje de analfabetismo, índice de marginación, índice de desarrollo humano, proporción de municipios no marginados en la entidad 